\title{
LHomme
}

L'HOMME Revue française d'anthropologie

$213 \mid 2015$

Varia

\section{Geneviève Calame-Griaule (1924-2013)}

Éric Jolly

\section{OpenEdition}

\section{Journals}

Édition électronique

URL : http://journals.openedition.org/lhomme/23653

DOI : 10.4000//homme.23653

ISSN : 1953-8103

\section{Éditeur}

Éditions de l'EHESS

\section{Édition imprimée}

Date de publication : 26 février 2015

Pagination : 7-18

ISSN : 0439-4216

\section{Référence électronique}

Éric Jolly, « Geneviève Calame-Griaule (1924-2013) », L'Homme [En ligne], 213 | 2015, mis en ligne le 25 février 2017, consulté le 03 mai 2019. URL : http://journals.openedition.org//homme/23653 ; DOI

10.4000/lhomme.23653

(c) École des hautes études en sciences sociales 


\section{Geneviève Calame-Griaule (1924-2013)}

Éric Jolly

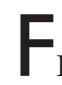

ILLE AÎNÉE de l'ethnologue Marcel Griaule, Geneviève Calame-Griaule a suivi la voie tracée par son père en menant des recherches en pays dogon dès l'âge de vingt et un ans, mais elle n'en a pas moins produit une œuvre pionnière dans les domaines de l'ethnolinguistique et de la littérature orale. Sortir de l'ombre du père n'avait pourtant rien d'évident : ce chercheur charismatique n'a pas seulement entraîné sa fille sur son terrain ; il l'a initiée à l'ethnologie, l'a confiée à ses propres informateurs et a influencé certains de ses choix. En outre, Geneviève Calame-Griaule lui vouait une telle admiration qu'elle n'a jamais cessé de promouvoir ou de défendre ses travaux, alors qu'elle mettait elle-même l'accent sur plusieurs angles morts des études de Griaule ou de ses collaboratrices: la langue, l'analyse des récits oraux (leur contenu comme leur contexte d'énonciation) et les rapports entre langage, attitudes corporelles et comportements sociaux. Rendre compte de l'originalité et de l'intérêt toujours actuel de son travail revient donc en partie à montrer comment Geneviève Calame-Griaule a réussi à dépasser les présupposés de l'école Griaule, grâce à sa formation de grammairienne, à l'influence de ses professeurs de l'époque et à son désintérêt pour une cosmogonie dogon déconnectée des faits de langage et de la réalité sociale.

Quelques années avant sa disparition, Geneviève Calame-Griaule publie un texte émouvant sur ses souvenirs de la mission Dakar-Djibouti, expédition ethnographique transafricaine qui la sépara de son père pendant deux ans, de 1931 à 1933, alors qu'elle n'avait que six ans au moment de son départ (2009a). Elle rapporte notamment que dès cette époque, Marcel Griaule l'avait choisie pour le seconder dans ses recherches 
ethnologiques, sans doute en raison de son statut d'ainnée ${ }^{1}$ et de son goût pour l'étude. Dans une lettre de 1932 reproduite par sa fille (Ibid. : 291), il évoque d'ailleurs, avec tendresse, leurs futures missions communes. Ce choix précoce d'une filiation professionnelle n'est pas surprenant quand on connaît l'attachement de Griaule à son terrain et aux membres de son équipe. Quatorze ans plus tard, en septembre 1946, sa fille aînée le suit donc en pays dogon pour la première mission Griaule de l'après-guerre, après un diplôme de Lettres classiques. Pour pallier les lacunes linguistiques des missions précédentes, elle étudie systématiquement plusieurs parlers dogon en décrivant pour la première fois leur grammaire. Simultanément, elle transcrit et traduit une cinquantaine de contes, ou récupère les récits rapportés par écrit par un jeune Dogon lettré de dix-sept ans ${ }^{2}$.

À l'époque, ces contes n'avaient pas encore le statut d'objets de recherche et n'étaient donc pas commentés; ils lui servaient uniquement à enrichir son lexique et à saisir la syntaxe. En outre, Geneviève CalameGriaule précise elle-même que, pour ses débuts sur le terrain, les informateurs l'ont traitée comme une néophyte en lui transmettant majoritairement des fables pour enfants (Calame-Griaule, ed. 2006 : 19-20). Il faut toutefois relativiser cette auto-évaluation rétrospective qui doit beaucoup à la modestie de son auteure, car ce qui frappe à la lecture de ses premières notes de terrain ${ }^{3}$, c'est au contraire le professionnalisme de cette «débutante » qui, en trois mois, va tout à la fois décrire précisément la structure de la langue tor s s et de ses variantes, repérer une douzaine de dialectes différents (Calame-Griaule 1952), travailler sur les échanges de moqueries entre villages (Calame-Griaule 1954a), réunir un corpus impressionnant de contes, traduire de nombreuses prières rituelles à la demande des autres membres de la mission et, enfin, mener de petites enquêtes ethnographiques dans le prolongement de ses travaux sur la terminologie (par exemple dans les domaines vestimentaire ${ }^{4}$, musical, architectural ou métallurgique). Si les chants ou les récits populaires qu'elle recueille n'ont rien de "savants", ce corpus a justement le mérite de coller à la réalité

1. Geneviève Calame-Griaule était l'aînée d'une fratrie de trois sœurs.

2. Originaire du village d'Amani, Antemmeli Pudyugo avait été formé à l'école de la mission protestante. Geneviève Calame-Griaule a évoqué sa mission de 1946 dans plusieurs articles: cf., notamment, Geneviève Calame Griaule (2001), Geneviève Calame-Griaule \& Praline GayPara (2002: 10-15).

3. Les archives de Geneviève Calame-Griaule ont été déposées à la bibliothèque Éric-de-Dampierre (Laboratoire d'ethnologie et de sociologie comparative, CNRs-Université Paris Ouest Nanterre La Défense). Elles sont la source de la plupart des informations concernant ses notes de terrain ou ses cours.

4. Son premier article publié sera d'ailleurs consacré à la confection et à l'usage des vêtements dogon (Calame-Griaule 1951). 
observable et aux changements en cours, en se référant notamment au pouvoir des Blancs et à l'émigration salariale en Gold Coast, alors qu'au même moment Griaule est en quête d'une mythologie ou d'une cosmogonie dogon immuable et multiséculaire.

Après cette première expérience de terrain, Geneviève Calame-Griaule continue de brillantes études : elle obtient un diplôme d'arabe littéral à l'École des langues orientales en 1947, passe une licence d'arabe à la Sorbonne en 1948 et, l'année suivante, est reçue première à l'agrégation de grammaire. Entre 1949 et 1950, elle choisit d'enseigner les lettres au lycée, à Marrakech, puis à Troyes, devant des jeunes filles qui la vénéraient, selon le témoignage de l'une de ses élèves qui deviendra elle-même professeur de français (Carbonare 2012: 28-29). En 1951, elle entre au CNRS comme attachée de recherche agrégée et peut dès lors se consacrer pleinement à la recherche. En juillet 1953, elle profite de la présence à Paris du sergent dogon Koguem Dolo ${ }^{5}$, principal interprète de son père, pour enregistrer la fable du lièvre et de la hyène, mais aussi pour se faire expliquer ce récit et ceux recueillis en 1946. À l'issue de ce travail, elle publie sa première analyse de contes dogon en privilégiant une lecture mythologique, sur la base des enquêtes réalisées par les missions Griaule de 1950 et 1952 (Calame-Griaule 1954b). Elle reprend ainsi l'idée d'une cosmogonie fondatrice qui servirait de modèle implicite et de clé de compréhension à la société en général et à la littérature orale en particulier.

D'avril à mai 1954, elle repart avec son père à Sangha, en pays dogon, en emportant pour la première fois un magnétophone, ce qui lui permet d'enregistrer de multiples contes, chants, devinettes, devises, moqueries... Durant cette mission, elle interroge trois des principaux informateurs de Marcel Griaule sur les conceptions dogon de la parole (son origine mythique, son mécanisme, ses classifications symboliques, ses représentations graphiques). Poursuivie sur une dizaine d'années, cette recherche s'inscrit dans la continuité des travaux de Griaule sur la révélation mythique du langage, sur les signes, et sur les systèmes de classification ou de correspondances symboliques. Mais, à la différence de son père, Geneviève Calame-Griaule prend en compte les affects, la psychologie, les singularités individuelles sans jamais perdre de vue «la réalité sociale ni les comportements verbaux ", comme elle le précise elle-même (1981 : 10). Au lieu de se limiter à une poignée d'informateurs privilégiés - qui restituent des discours abstraits construits en interaction avec les enquêteurs -, elle observe comment les gens parlent et se conduisent dans la vie

5. Celui-ci était en transit en France avant de partir pour l'Indochine, où il sera blessé et capturé en janvier 1954, avant d'être libéré huit mois plus tard. 
courante. Elle enregistre d'ailleurs des conversations ordinaires entre des couples d'interlocuteurs variés : un homme et une femme, un adulte et un enfant, deux locuteurs de parlers différents, deux hommes, etc. Elle travaille aussi avec les femmes sur l'apprentissage linguistique de leurs enfants ou de leurs petits-enfants, tout en continuant ses enquêtes lexicographiques en prévision de la publication d'un dictionnaire dogon ${ }^{6}$.

Après la disparition prématurée de son père, en février 1956, Geneviève part à deux reprises en pays dogon avec son mari, le musicien Blaise Calame, afin d'explorer les liens entre parole et musique, de décembre 1956 à janvier 1957, et de novembre à décembre 1958. Pour ces deux missions, ils profitent de la vedette-laboratoire Mannogo ou de son équipement pour effectuer des enregistrements musicaux de qualité, le long du fleuve Niger, puis à Sangha. Lors de leur première mission commune, ils enregistrent près de dix heures de chants et de musique instrumentale variés, dont ils publieront des extraits, dans un disque intitulé Scènes de la vie des Dogon (1957b). Ensemble, ils se lancent également dans une vaste enquête sur le système musical des Dogon et sur leur conception de la musique en abordant notamment la sémantique, la valeur des voix et le rapport entre tons, hauteurs de notes et formules rythmiques ou mélodiques. En se plaçant au carrefour de la linguistique, de l'ethnologie, de la musicologie et de la psychologie, Geneviève CalameGriaule parvient, là encore, à faire dialoguer plusieurs disciplines pour saisir tous les aspects d'un même phénomène, musical ou langagier. Cette enquête originale, voire expérimentale, se poursuivra jusqu'à la cinquième mission de Geneviève Calame-Griaule, en novembre-décembre 1960 ; ses résultats serviront de matériaux à plusieurs articles et à un chapitre de sa thèse ${ }^{7}$. Méconnu et peu discuté, ce travail transdisciplinaire mériterait d'ailleurs d'être revisité par un historien des sciences, car il fait ressortir en creux l'absence de dialogue entre ethnolinguistes et ethnomusicologues français, en dépit de pratiques similaires et d'une même attention portée à la voix et aux sons.

Entre 1956 et 1960, Geneviève Calame-Griaule entreprend également la révision de son dictionnaire dogon tor $s$ s, enrichit son corpus de contes (en étendant sa collecte aux rives du Niger), effectue de nouvelles enquêtes lexicologiques sur des domaines précis (cuisine, outils, expression du temps...) et, enfin, complète ses travaux sur la parole proprement dite,

6. Ce Dictionnaire dogon, dialecte t̀̀r sera publié en 1968, après avoir été présenté comme thèse secondaire.

7. Cf. notamment Geneviève Calame-Griaule \& Blaise Calame (1957a) ; Geneviève CalameGriaule (1965: 526-543). 
en particulier lors de sa mission de 1960 , en travaillant notamment sur la gestuelle et les postures des locuteurs, ou encore en étudiant l'échange verbal dans le cadre de disputes, de demande de pardon, ou de diffamation.

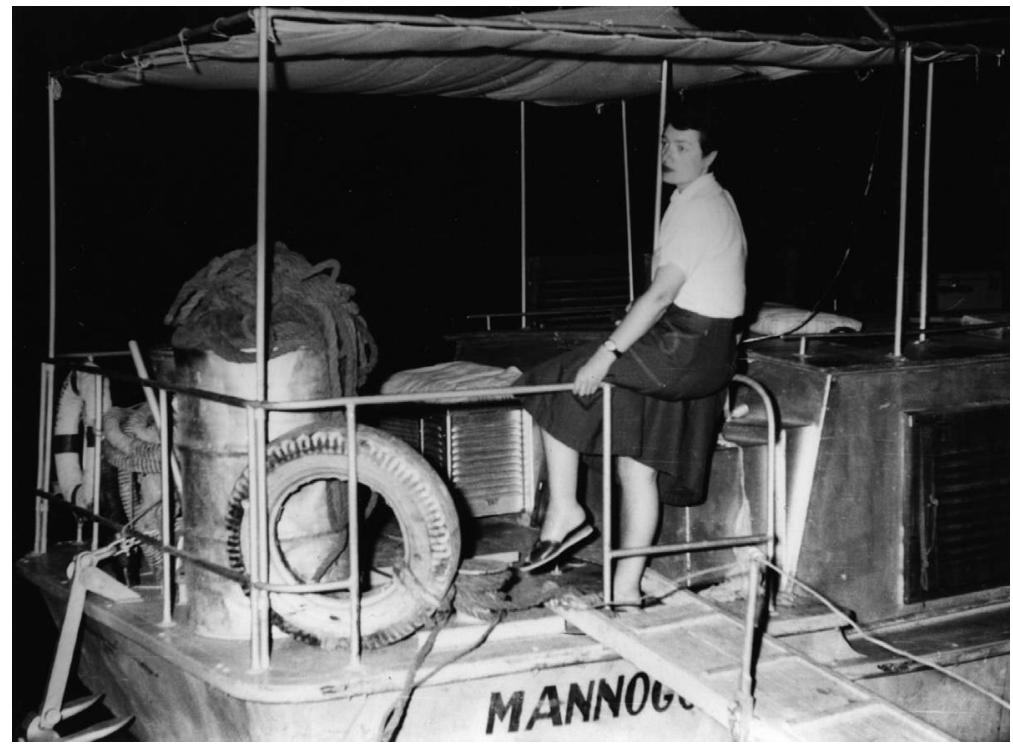

Geneviève Calame-Griaule sur le Mannogo, Soudan français, 1956.

Cliché Blaise Calame. Fonds Geneviève-Calame-Griaule,

Bibliothèque Éric-de-Dampierre, MAE, Université Paris Ouest-Nanterre-La Défense.

Parallèlement, elle assiste régulièrement aux cours d'Émile Benveniste, de 1954 à 1964, avant de suivre les enseignements d'André Martinet, de 1963 à 1966. Comme le suggèrent ses notes de cours, ces deux linguistes ont eu sur elle une influence déterminante : le premier, en insistant sur le rôle "de la subjectivité dans le langage" et sur l'implication des faits linguistiques dans l'ensemble des activités humaines (Benveniste 1966); le second, en soulignant la fonction de communication des gestes, des tons, de la prosodie et de la stylistique (Martinet 1960). Par ailleurs, entre 1960 et 1964, elle suit les cours et les séminaires de Claude Lévi-Strauss (Équipe "Langage et culture en Afrique de l'Ouest» 1989: 424) et s'en inspire pour donner une orientation structurale à ses analyses de contes et à sa classification des paroles, en mettant notamment l'accent sur les oppositions binaires qui régissent, selon elle, la vision du monde des Dogon. D’après son propre témoignage, elle se nourrit également de la lecture des ouvrages de Marcel Granet (1934), de Maurice Leenhardt (1947) et de Marcel Cohen (1956) ${ }^{8}$.

8. Sur l'influence de ces travaux, cf. Geneviève Calame-Griaule (1977a : 13-14, 2009b : 564). 
Soutenue en 1966, la thèse principale de Geneviève Calame-Griaule est le résultat le plus marquant de l'ensemble des travaux de terrain, des enseignements et des lectures déjà mentionnés. Une version en avait été publiée en 1965, chez Gallimard sous le titre Ethnologie et langage. La parole chez les Dogon ${ }^{9}$, étude fondatrice sur les rapports entre langage et société qui ouvrira la voie aux recherches "ethnolinguistiques» en France, alors que les travaux similaires de l'Américain Dell Hymes ${ }^{10}$ sont encore inconnus des universitaires français. Centrée sur la conception dogon de la parole, la première moitié du livre complète en partie les explications mythologiques, symboliques et graphiques publiées précédemment par Marcel Griaule, mais en leur donnant une coloration plus psychologique et structurale, sous l'influence conjointe de la psychanalyse et du structuralisme. L'auteure se distingue également des travaux de l'école Griaule par sa prise en compte des représentations populaires, par la finesse de ses analyses sémantiques, et par une vision nettement moins figée des classifications, des mythes ou des graphies dogon, qu'elle présente plutôt comme des exemples significatifs et non comme des éléments immuables d'un système qui existeraient indépendamment du contexte de l'enquête et de l'identité du locuteur ou du dessinateur ${ }^{11}$.

La partie du livre la plus originale et la plus représentative de l'œuvre de Geneviève Calame-Griaule est celle où l'auteure étudie les échanges verbaux de la vie courante, les mécanismes d'apprentissages linguistiques, les expressions sonores des émotions et les différents usages de la parole. Attentive aux faux-semblants et aux effets de sens produits par les situations discursives, elle rend compte du caractère factice des secrets des hommes ou des femmes, du décalage important entre discours normatifs et points de vue réels, ou encore de l'importance des procédés stylistiques et du contexte d'énonciation pour saisir le sens, l'intention et l'efficacité d'une prière, d'un serment, d'une malédiction ou d'une insulte. Croisant analyses structurales et approche ethnolinguistique, elle aborde les contes ou les chants en étudiant leurs significations, leurs règles, leurs variantes, leurs évolutions et leur dimension performative. Toutes ces analyses restent pertinentes un demi-siècle plus tard et renouvellent autant les travaux sur

9. Sur ce livre, l'analyse qui suit s'inspire d'un compte rendu de sa troisième édition, paru dans les Cahiers d'études africaines (Jolly 2013).

10. Ce fondateur de l'ethnographie de la communication (Hymes 1962) signera en 1986 la préface de l'édition américaine d'Ethnologie et langage. Le texte sera repris dans la troisième édition française (Hymes 2009).

11. Geneviève Calame-Griaule n'hésite pas, d'ailleurs, à reproduire côte à côte des dessins représentant de façon radicalement différente une même étape de la parole, alors que ces versions divergentes - graphiques ou mythologiques - sont systématiquement gommées dans le livre publié, la même année, sous la double signature de Marcel Griaule et Germaine Dieterlen (1965). 
la littérature orale que les études sur les Dogon. Au lieu de promouvoir l'image d'une tradition orale invariable et d'une société figée, sans conflits ni individualités, Ethnologie et langage souligne au contraire l'inventivité des chanteurs, la variabilité des récits et la diversité des choix individuels, sans occulter l'influence de l'islam, le contexte colonial, les situations de conflits et d'affrontements violents. À cette époque, une approche plus politique de la parole et de la littérature orale faisait encore défaut, mais, quelques années plus tard, Geneviève Calame-Griaule comblera cette lacune en proposant une lecture politique de plusieurs contes dogon ou africains ${ }^{12}$.

Après sa soutenance, Calame-Griaule retourne à quatre reprises en pays dogon : en novembre 1967, en février-mars 1969, en novembre 1970 et en décembre 1983. Durant les trois premières missions, elle mène une étude exhaustive sur les serrures en bois, dans le prolongement de ses recherches sur les rapports entre parole et expressions plastiques ${ }^{13}$. Pendant cette période, elle travaille sur la " langue du sigui » à la demande de Germaine Dieterlen et Jean Rouch. Ces derniers ont filmé dans la région de Sangha le rituel soixantenaire du sigui et ont besoin de traduire les discours prononcés dans ce langage exclusivement masculin. Jamais publiées, cette enquête de grande ampleur et ces traductions au service des autres témoignent de la générosité de leur auteure. Elles valident également les recherches et les analyses antérieures de Michel Leiris (1948). Les quatre nouvelles missions de Geneviève Calame-Griaule en pays dogon servent enfin à compléter son corpus de contes et à recueillir des commentaires permettant d'analyser les récits collectés, à l'image de l'enquête de 1983 sur le miel, dans la perspective d'un article sur "Le miel des relations humaines" (1985). À la suite de ces nouvelles recherches de terrain, on peut noter plusieurs inflexions notables dans les publications de Geneviève Calame-Griaule sur les contes dogon: ses analyses privilégient de plus en plus le schéma structural du récit, son interprétation initiatique ${ }^{14}$ et un comparatisme typologique qui s'inspire de Lévi-Strauss et de la classification d'Aarne et Thompson ${ }^{15}$. Sans être nouveau, le recours à la psychanalyse - pour cerner les motifs œdipiens ou sexuels qui sous-tendent les contes - devient plus explicite (CalameGriaule \& Lacroix 1970 ; Calame-Griaule 1972).

12. Cf., par exemple, Geneviève Calame-Griaule \& Veronika Görög-Karady (1972 : 48-52).

13. Le résultat de ce travail sera publié sous forme de microfiches (Calame-Griaule, Dupuis \& Ndiaye 1976).

14. Un grand nombre de contes «initiatiques » africains analysés par Geneviève Calame-Griaule seront rassemblés et republiés dans le recueil intitulé Des Cauris au marché (1987).

15. Cf., par exemple, Geneviève Calame-Griaule (1969, 1976). 
Mais ces études, aussi brillantes soient-elles, ne correspondent pas à la dimension la plus novatrice des travaux de Geneviève Calame-Griaule, si on les compare à ses analyses sur la performance orale des conteurs. En 1971, elle transcrit un conte dogon en décrivant minutieusement, pour chaque phrase, les procédés expressifs employés par le conteur (intonation, répétitions, choix des mots, construction des phrases...). Elle démontre ainsi que toute performance donne en partie sens au récit, en renouvelant à chaque fois sa forme artistique. La variabilité des récits et leur réception sont donc directement liées au style, à la créativité et au talent propres à chaque locuteur (Calame-Griaule 1971) ${ }^{16}$. Une telle conclusion, qui nous paraît aujourd'hui évidente, éloigne encore un peu plus Geneviève Calame-Griaule d'une école Griaule qui, après-guerre, refuse d'interroger et de reproduire la diversité des discours afin d'obtenir, par sélection ou par synthèse, un récit unique et univoque conforme à ses attentes.

Dans les années qui suivent, Geneviève Calame-Griaule poursuit ses travaux sur la performance orale, mais sur un nouveau terrain et sur un sujet de recherche quasiment inexploré : la gestuelle des conteurs. Entre 1967 et 1978, elle effectue six missions au Niger, dans les environs d'Agadez, chez les Touaregs et les Isawaghen ${ }^{17}$. Dès son deuxième séjour, en octobre 1970, elle enregistre, photographie et transcrit les performances des conteurs - en majorité des femmes ou des forgerons - en notant séparément, sur deux pages placées en vis-à-vis, le texte et ses observations (sur les mimiques, les gestes et les intonations). En 1977, un article présente les résultats de cette recherche inédite sur les gestes narratifs " en analysant un texte touareg du point de vue des procédés expressifs employés par le narrateur»(Calame-Griaule 1977b : 309). Une telle étude montre notamment que la gestuelle du conteur a tout à la fois une valeur mnémotechnique, phatique, informative, explicative et théâtrale. En 1975 et 1978, Geneviève Calame-Griaule complète ses méthodes d'enregistrement du geste grâce à la caméra d'Edmond Bernus ${ }^{18}$, qui

16. Juste avant cette étude de cas, dans un article plus général définissant pour la première fois l'ethnolinguistique, Geneviève Calame-Griaule insistait déjà sur la créativité et la liberté stylistique de chaque récitant ou narrateur africain en concluant : «Restituer les textes dans leur intégralité implique de considérer chacun d'eux comme un message unique et original, produit dans un contexte culturel particulier, par l'intermédiaire d'une langue et d'un agent dont il est inséparable» (1970 : 44).

17. Pour ses missions de 1967 et 1970, Geneviève Calame-Griaule combine terrain nigérien (en octobre) et terrain dogon (en novembre).

18. Dans son hommage au géographe Edmond Bernus, Geneviève Calame-Griaule (2005) revient sur leur collaboration au sein d'une RCP (Recherche coopérative sur projet) dont faisaient également partie l'ethnologue Suzy Bernus et le linguiste Pierre-Francis Lacroix. 
travaille sur le même terrain. Et au terme de cette enquête ${ }^{19}$, elle rassemble dans un même recueil le répertoire de plusieurs conteurs isawaghen dont elle dresse un portrait vivant et chaleureux, en vantant la variété de leurs talents (Calame-Griaule 2002).

Son premier article sur les gestes narratifs est publié dans un ouvrage collectif qu'elle coordonne et qui va servir de manifeste à l'ethnolinguistique "africaniste": Langage et cultures africaines. Essais d'ethnolinguistique (1977). Dix ans plus tard, Geneviève Calame-Griaule dirige également un numéro spécial du Journal des africanistes consacré aux Voix de la parole (1987) - dont les seize contributions témoignent non seulement de la vitalité des études ethnolinguistiques sur l'Afrique, mais aussi de leur ouverture à des axes de recherche relativement nouveaux pour la discipline (du rapport entre oralité et écriture aux relations de pouvoir). Sur les contes, Geneviève Calame-Griaule organise plusieurs colloques ou journées d'études qui donneront lieu à deux publications couvrant toutes les aires culturelles : la première fait le point sur les différentes méthodes d'analyse des contes (Calame-Griaule, Chiche \& Görög-Karady, eds 1984), tandis que la seconde, plus marquante peut-être, rend compte du renouveau du conte oral dans les pays européens et américains, avec l'apparition de néo-conteurs professionnels ou semi-professionnels (Calame-Griaule, ed. 1991). Dans ce livre collectif, qui témoigne de l'attention de Geneviève Calame-Griaule aux évolutions contemporaines, les contributeurs transmettent leur expérience, interrogent la relation entre écrit et oral, analysent la transformation des répertoires ou des styles et examinent les nouvelles fonctions du conte.

Les responsabilités importantes assumées par Geneviève CalameGriaule dans les domaines de l'édition, de l'enseignement et de l'administration de la recherche ont contribué également à la reconnaissance et au développement d'un champ disciplinaire qu'elle n'a jamais cessé de promouvoir, en entraînant dans son sillage plusieurs générations de chercheurs français et africains. Entre 1967 et 1987, elle dispense des cours d'ethnolinguistique et de littérature orale à l'EPHE, à l'INALCO ou à l'Université Paris III. En 1976, elle participe à la création des Cahiers de littérature orale, dont elle deviendra directrice de la publication. Un an plus tard, elle prend la direction de l'équipe «Langage et culture en Afrique de l'Ouest » (CNRS-INALCO) ; et en 1998, elle préside l'association des Classiques africains qui publie en édition bilingue des œuvres de la littérature orale africaine.

19. Pour une description des enquêtes sur la gestuelle des conteurs nigériens, cf. Geneviève Calame-Griaule (2008). 
Cette rapide reconstitution du parcours scientifique et académique de Geneviève Calame-Griaule manquerait de sensibilité s'il n'évoquait pas, pour finir, la générosité et la passion qui l'animaient, source d'admiration unanime pour ses étudiants et ses collègues.

Centre national de la recherche scientifique Institut des mondes africains (IMAf), Ivry-sur-Seine eric.jolly@cnrs.fr

\section{BIBLIOGRAPHIE SÉLECTIVE DE GENEVIÈVE CALAME-GRIAULE}

\section{Calame-Griaule, Geneviève}

1951 «Le vêtement dogon, confection et usage ", Journal de la Société des africanistes 21 (2) : 151-163.

1952 "Diversité et organisation sociale chez les Dogon du Soudan français ", Notes africaines 55 : 77-79.

1954a «Les moqueries de villages au Soudan français ", Notes africaines 61 : 12-15.

1954 b "Ésotérisme et fabulation au Soudan ", Bulletin de l'IFAN 16 (3-4) : 307-321.

1965 Ethnologie et langage. La parole chez les Dogon. Paris, Gallimard.

1968 Dictionnaire dogon, dialecte tor $\mathrm{s}$. Langue et civilisation. Paris, Librairie Klincksieck («Langues et littératures de l'Afrique noire").

1969 "L'arbre au trésor ", in G. CalameGriaule, ed., Le Thème de l'arbre dans les contes africains. Paris, Sélaf: 25-58.

1970 "Pour une étude ethnolinguistique des littératures orales africaines ", Langages 18 : L'ethnolinguistique : 22-47

[http://www.persee.fr/web/revues/home/ prescript/article/lgge_0458-

726x_1970_num_5_18_2026].

1971 «Étude stylistique d'un conte dogon ", in Veronica Six et al., eds, Afrikanische Sprachen und Kulturen. Ein Querschnitt. Hamburg, Deutsches Institut für Afrika-Forschung : 266-278.
1972 «Une affaire de famille : réflexions sur quelques thèmes de "cannibalisme" dans les contes africains ", Nouvelle Revue de psychanalyse 6 : 171-202.

1976 «La calebasse brisée : étude du thème initiatique dans quelques versions africaines des "Deux Filles" (AT 480)», Cahiers de littérature orale $1: 23-66$.

1977a «Introduction : pourquoi l'ethnolinguistique ", in G. Calame-Griaule, ed., Langage et cultures africaines... : 11-28.

1977 b "Pour une étude des gestes narratifs", in G. Calame-Griaule, ed., Langage et cultures africaines... : 303-359.

1981 «Délimitation d'un terrain en ethnolinguistique : la parole chez les Dogon ", suppl. Langage et société 17 : 9-11.

1985 "Le miel des relations humaines", Cahiers de littérature orale $18: 65-85$.

1987 Des cauris au marché. Paris, Mémoires de la Société des africanistes.

2001 "Avec Germaine en pays dogon (1946) ", Journal des africanistes 71 (1): 35-51.

2002 Contes tendres, contes cruels du Sahel nigérien. Paris, Gallimard.

2005 «Edmond Bernus (1929-2004)", Journal des africanistes 75 (1) : 295-299.

2008 «Dites-le avec des gestes : comment étudier la gestuelle des conteurs?", Cahiers de littérature orale 63-64:83-108. 
2009a «Le temps de l'absence », in Nicolás Sánchez Durá

\& Hasan G. López Sanz, eds, La Misión etnográfica y lingüistica Dakar-Djibouti y el fantasma de África (1931-1933).

Valencià, Museu Valencià de la Illustraciò i de la Modernitat: 289-291.

2009b "Postface», in G. Calame-Griaule, Ethnologie et langage. La parole chez les Dogon. Limoges, Lambert Lucas: 563-572.

\section{Calame-Griaule, Geneviève, ed.}

1977 Langage et cultures africaines. Essais d'ethnolinguistique. Paris, François Maspero ( Bibliothèque d'anthropologie »).

1987 Journal des africanistes 57 (1-2) :

Les Voix de la parole: 7-16.

1991 Le Renouveau du conte.

Paris, Éd. du CNRS.

2006 Contes dogon du Mali.

Paris, Karthala-Langues O', Inalco

(« Paroles en miroir»).

\section{Calame-Griaule, Geneviève}

\section{\& Blaise Calame}

1957a «Introduction à l'étude de la musique africaine ", La Revue musicale 238 : Carnets critiques.

1957b Les Trésors de l'art musical populaire. Scènes de la vie des Dogon (Soudan français). Enregistrement sonore. S.l., Résonances. Disque 33t.
Calame-Griaule, Geneviève, Michèle Chiche \& Veronika Görög-Karady, eds

1984 Le Conte, pourquoi? Comment? d'étude en littérature orale "Analyse des contes, problèmes de méthodes", Paris, 23-26 mars 1982. Paris, Éd. du CNRS ("Colloques internationaux du CNRS »).

Calame-Griaule, Geneviève, Annie Dupuis \& Francine Ndiaye, eds

1976 Serrures dogon. Analyse ethnomorphologique. Paris, Institut d'ethnologie.

Calame-Griaule, Geneviève

\& Praline Gay-Para

2002 La Parole du monde. Parole, mythologie et contes en pays dogon. Entretiens. Paris, Mercure de France («Le Petit Mercure»).

Calame-Griaule, Geneviève \& Veronika Görög-Karady

1972 « La calebasse et le fouet: le thème des "objets magiques" en Afrique occidentale", Cahiers d'études africaines 45 : 12-75.

Calame-Griaule, Geneviève \& Pierre-Francis Lacroix

1970 "La "mère vendue" : essai d'analyse d'un thème de conte africain ", in Pierre Maranda \& Jean Pouillon, eds, Échanges et communications. Mélanges offerts à Claude Lévi-Strauss à l'occasion de son $60^{\circ}$ anniversaire. La Haye-Paris, Mouton : 1356-1380 ("Studies in General Anthropology»). 
Benveniste, Émile

1966 Problèmes de linguistique générale, 1. Paris, Gallimard.

\section{Carbonare, Marguerite}

2012 Fracture et souffle de vie.

Paris, L'Harmattan.

Cohen, Marcel

1956 Pour une sociologie du langage. Paris, Albin Michel.

Équipe "Langage et culture en Afrique de l'Ouest", ed.

1989 "Profil d'une recherche", Graines de paroles. Puissance du verbe et traditions orales. Textes offerts à Geneviève Calame-Griaule. Paris, Éd. du CNRS : 421-427.

\section{Granet, Marcel}

1934 La Pensée chinoise.

Paris, Renaissance du livre-Albin Michel ( L'Évolution de l'humanité»).

\section{Griaule, Marcel \& Germaine Dieterlen}

1965 Le Renard pâle.

Paris, Institut d'ethnologie.

\section{Hymes, Dell H.}

1962 "The Ethnography of Speaking", in T. Gladwin \& W. C. Sturtevant, eds, Anthropology and Human Behavior. Washington, Anthropological Society of Washington : 13-53.

2009 «Préface de l'édition américaine (1986) ", in G. Calame-Griaule, Ethnologie et langage. La parole chez les Dogon... : XI-XIV.

Jolly, Éric

2013 «Compte rendu du livre de Geneviève Calame-Griaule, Ethnologie et langage. La parole chez les Dogon. Troisième édition revue et corrigée, Limoges, LambertLucas, 2009 ", Cahiers d'études africaines $211: 735-737$.

\section{Leenhardt, Maurice}

1947 Do Kamo. La personne et le mythe dans le monde mélanésien. Paris, Gallimard.

Leiris, Michel

1948 Langue secrète des Dogons de Sanga (Soudan français). Paris, Institut d'ethnologie ("Travaux et mémoires de l'Institut d'ethnologie »).

Martinet, André

1960 Éléments de linguistique générale. Paris, Armand Colin. 